\title{
Introduction of ultrasound at the bedside in the differential diagnosis of the pulmonary embolism as a cause of syncope
}

\author{
Ion Koldobika Iríbar Diéguez ${ }^{*}$, Enrique Bello Larrarte²
}

\author{
* Correspondence \\ Ion Koldobika Iribar Diéguez \\ Emergency Department. Bidasoa \\ Hospital. Finca Zubieta s / n 20280. \\ Hondarribia (Guipúzcoa, Spain) \\ Email: ionkiribar@yahoo.es \\ Telephone: 696.700052/943-007700 (Ext \\ 2707) \\ 'Emergency Department of the Bidasoa \\ OSI-Hospital of Hondarribia \\ 2Radiology Department of the Bidasoa \\ OSI-Hospital of Hondarribia \\ Received: Jan 14, 2019 \\ Accepted: Feb 21, 2019 \\ Published: Feb 23, 2019
}

\begin{abstract}
There is currently no emergency congress without a table and/ or ultrasonography workshop at the bedside and recently the new guidance on the management of syncope has been published (ESC Guide 2018 on the diagnosis and treatment of syncope [1]). Together with the possibility of cushioning the problem of the excess of requests for complementary tests in the emergency departments, we believe that this article combines both aspects and provides an interesting point of reflection.
\end{abstract}

Keywords: Syncope, Pulmonary embolism, Ultrasound at the bedside, Supplementary tests.

\section{Introduction}

Syncope accounts for $1-3 \%$ of the consultations in the emergency room, and the estimate of syncope caused by a pulmonary embolism (PE) is low (0.8-1\%) [2]. Conversely, $10 \%$ of the PE present with syncope [3] .

The classic diagnostic algorithm of syncope involves: anamnesis, physical examination, orthostatism test, carotid sinus massage, risk stratification, ECG, and analytical (including D-dimer if there is clinical and/ or suspicious signs of PE) [1]. Thus, the percentage of normal angioTAC derived from obtaining a high Ddimer (DD) in cases of syncope is: $74-88 \%$ [4]. This implies: poor profitability of DD $(500 \mathrm{ng} / \mathrm{mL})$ [4]: specificity $45 \%$ and sensitivity $94 \%$; and the realization of an unnecessary number of angioTAC, with its complications: radiation, contrast allergies, deterioration of renal function, diagnosis of noncritical PE with consequent anticoagulation and its risks.

Until now, coherently, whenever the situation was not suspicious, we obviated requesting this type of analysis with low profitability and associated complications.

However occasionally we find cases of syncope caused by a critical oligosymptomatic PE ( $50 \%$ of the PE can be silent, without deep venous thrombosis [5] $)$.

\section{Clinical cases}

Case 1: Woman 70 years old, hypertensive. Syncope when getting out of bed, without prodrome, without chest pain, palpitations or dyspnea. Hemodynamically stable (FC 75 lpm). General, cardiovascular and neurological examination: normal. OESIL 2. Wells 1 / Geneva 3. 2nd syncope in the emergency room after 7 hours of normal monitoring. Massive PE. Death.

Case 2: Woman 46 years old, healthy. Syncope while ironing, without prodromes, without chest pain, without palpitations or dyspnea. Hemodynamically stable at rest (HR 104 bpm). General, cardiovascular and neurological examination: normal. OESIL 1. Wells 1 / Geneva 5. Massive bilateral PE. Lysis of the thrombus. 


\section{Discussion}

Given this situation, we consider the need to diagnose these cases without having to assume the poor profitability of DD and its negative consequences. We suggest the use of ultrasound at the door of the bed in the emergency departments during the diagnosis of syncope could give us that possibility, as defended by different authors such as Squizzato A [], Abassi OZ [ㅁ] or Chung-Esaki H []].

We know that only a massive PE produces syncope [ $\underline{2}$, 6], and also that a critical PE produces pulmonary hypertension and dilation of right cardiac cavities [ $\underline{5}$, 7]. Therefore, if we perform an ultrasound at the foot of the bed (non-invasive and without intrinsic complications) on the patient with syncope and discard right ventricle dilatation, we will be sure of having eliminated a PE as the cause of syncope, without the need for further complementary tests (Figure 1). With this combined diagnostic approach to syncope, we will maintain DD> $90 \%$ sensitivity and increase its specificity to $86 \%$ [5].

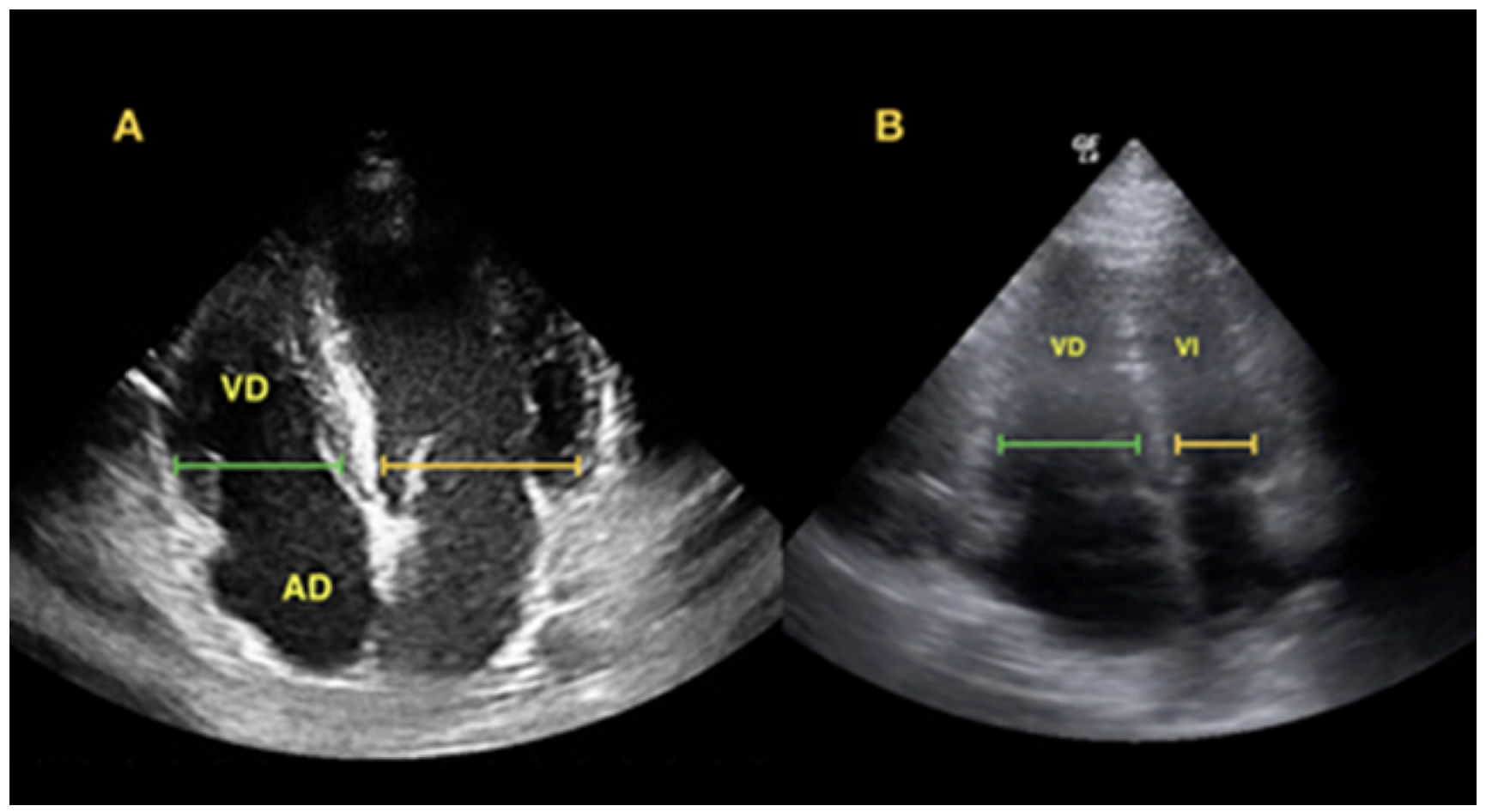

Figure 1: Measurement of the right ventricle (RV) in a normal individual (A) and in a patient with RV dilation secondary to lung embolism (B). Image obtained from the basic echocardiography course for non-cardiologists.

Therefore, it is advised that the introduction of ultrasound at the bedside within the diagnostic scheme of syncope is useful to exclude the diagnosis of critical PE (in addition to ruling out other causes such as cardiac tamponade) and, although this approach is not diagnostic definitively, it improves the profitability of DD in order to obtain further confirmatory imaging tests. Ultrasound has limitations and therefore, in no case replaces the angioTAC as a modality for definite diagnosis. MRI could open up an avenue in this aspect. However, the technical limitations and the limited application in situations of urgency are its main drawbacks [8].

\section{Conclusion}

With this evaluation of clinical cases and disquisition on the contributions of ultrasound at the bedside in cases of syncope, we seek to generate debate and studies that can demonstrate the advantages of its use in the emergency services in these cases.

\section{Conflict of interest}

- The work does not present a conflict of interest

- The work has received no financing

- The work has been partially reported through oral communication within the XXIII EKALME-SEMES Euskad Emergencies Conference. 


\section{Copyrights}

(c) Ion K I Diéguez et al, 2019; licensee OA Journal of Clinical Case Reports. This is an Open Access article distributed under the terms of the Creative Commons Attribution License (http:// creativecommons.org/licenses/by/4.0), which permits unrestricted use, distribution, and reproduction in any medium, provided the original work is properly credited.

\section{References}

1. Brignole M, Moya Á, Lange F.J.D, et al. (2018) Grupo de Trabajo de la Sociedad Europea de Cardiología (ESC) para el diagnóstico y tratamiento del síncope. Rev Esp Cardiol. 71:e1e92.

2. Oqab Z, Ganshorn H, Sheldon R. (2017) Prevalence of pulmonary embolism in patients presenting with syncope. A systematic review and meta-analysis. Am J Emerg Med. 36:551-555.

3. Gutierrez J.J.Q, Gordillo M.A.M, Naon A.L, et al. (2005) Mujer joven con síncope y tromboembolismo pulmonar bilateral. Emergencias. 17:152-153.
4. Royo N.L, Arri E.A, Montero A.G, et al. (2014) Probabilidad clínica de tromboembolismo pulmonar: beneficio diagnóstico de las escalas de predicción y de los dímeros D. Emergencias. 26: 243-250.

5. Squizzato A, Galli L, Gerdes V.E.A. (2015) Point-of-care ultrasound in the diagnosis of pulmonary embolism. Crit Ultrasound J. 7:7.

6. Abbasi OZ, Doan TT, Duggal S, et al. (2016) Utilization of bedside ultrasound in the diagnosis and management of massive pulmonary embolism: a case report. Radiol Case Rep. 11:447-449.

7. Chung-Esaki H, Knight R, Noble J, et al. (2012) Detection of Acute Pulmonary Embolism by Bedside Ultrasound in a Patient Presenting in PEA Arrest: A Case Report. Case Rep Emerg Med. 2012:794019.

8. Hochhegger B, Ley-Zaporozhan J, Marchiori E, et al. (2011) Magnetic resonance imaging findings in acute pulmonary embolism. Br J Radiol. 84:282-287. 